\title{
Cross-Layer Analysis of Joint Rate and Power Adaptation in Nakagami Fading Channels with Multiple-User Contention
}

\author{
Li-Chun Wang · Jane-Hwa Huang • Anderson Chen • \\ Chung-Ju Chang
}

Published online: 10 July 2009

(C) Springer Science+Business Media, LLC. 2009

\begin{abstract}
Adaptively adjusting transmit rate and power concurrently to enhance goodput and save energy is a challenging issue in a wireless local area network (WLAN) because goodput enhancement and energy saving are usually two contradictory goals. In this paper, we propose channel-driven rate and power adaptation (CDRPA) schemes and develop a physical (PHY)/medium access control (MAC) cross-layer analytical method incorporating the impacts of Nakagami fading channel and the carrier sense multiple access (CSMA) MAC protocol. The CDRPA scheme has much lower computation complexity than the energyoptimal complete-search scheme. In a multiuser contention scenario, we analyze the energy efficiency and the goodput of the power-first and rate-first CDRPA schemes as well as the energy-optimal complete-search adaptation scheme. At the cost of lower goodput, the powerfirst scheme has better energy efficiency than the rate-first CDRPA scheme, whereas if the goodput is the main concern, the rate-first CDRPA scheme shall be chosen due to better goodput performance. More interestingly, we find that the power-first CDRPA scheme can achieve about the same goodput and energy efficiency as the energy-optimal complete-search link adaptation scheme.
\end{abstract}

Keywords Wireless local area network (WLAN) · Rate and power adaptation · Cross-layer analysis

L.-C. Wang (ه) · J.-H. Huang · C.-J. Chang

Department of Communication Engineering, National Chiao Tung University,

Hsinchu 300, Taiwan, ROC

e-mail: lichun@cc.nctu.edu.tw

J.-H. Huang

e-mail: hjh@mail.nctu.edu.tw

C.-J. Chang

e-mail: cjchang@cc.nctu.edu.tw

A. Chen

Information \& Communications Research Laboratories, Industrial Technology Research Institute of Taiwan, Hsinchu 300, Taiwan, ROC

e-mail: mbchen@itri.org.tw 


\section{Introduction}

Rate adaptation and power control are important techniques for wireless networks to enhance goodput and save energy. Ideally, by dynamically adapting the transmission parameters (e.g., transmit power level, modulation and coding scheme) to the channel quality, rate adaptation can help enhance channel capacity and power control can help lower energy consumption. However, goodput enhancement and energy saving are indeed two contradictory goals. To achieve higher goodput, it is necessary to operate at a higher level of modulation with fewer redundant bits in coding, thereby requiring higher transmit power to maintain acceptable bit error rate (BER) performance. Therefore, how to adaptively adjusting transmit rate and power to concurrently enhance goodput and save energy is a difficult challenge in wireless networks.

In the literature, the rate and power adaptation schemes are designed separately for improving either goodput or energy efficiency. To enhance goodput, an auto rate fallback (ARF) adaptation scheme was proposed in [1]. In the ARF scheme, the user has to count the number of successfully received and missing acknowledgement (ACK) frames. If consecutively failing to receive two ACK frames, the user lowers the data rate in the next retransmission. If successfully receiving ten ACK frames, the user can raise the data rate for the next frame transmission. In [2], the rate adaptation scheme is based on the long-term and shortterm statistics of successful and failed transmitted frames. Clearly, these algorithms in [1] and [2] take a long time to count the number of successfully received ACK frames, and thus may not react readily to the fluctuations of wireless channel. Therefore, many studies [3-7] focused on developing fast rate adaptation algorithms without counting the number of successive successful and failed transmissions. The adaptation schemes in $[3,4]$ made use of the request-to-send/clear-to-send (RTS/CTS) handshaking to estimate and feedback the channel quality. Then, the user adjusts the data rate according to the SNR estimation. Since the CTS frame format should be modified to include the SNR information, the methods in $[3,4]$ are not compatible with the existing wireless local area networks (WLANs). In [5], a goodput-optimal link adaptation scheme was proposed to select the modulation and coding scheme (MCS), aiming at maximizing the goodput in the additive white Gaussian noise (AWGN) channel. Generally, the MCS selection $(m)$ depends on the frame payload $(l)$, the received signal strength $(s)$, and the retry count $(n)$. By exhaustively searching among all the combinations of $(m, l, s, n)$, the scheme in [5] selects the one with the highest goodput. With a complete search method, this approach can achieve the optimum goodput performance in the AWGN channel. However, the computation complexity of searching all the combinations of $(m, l, s, n)$ is a concern from the power saving viewpoint. In addition, the optimal choice of $(m, l, s, n)$ in the AWGN channel may be unsuitable for the multipath fading channel. To decrease the computation complexity, the authors [6-8] suggested a reduced PHY mode table for rate adaptation by removing some energy-inefficient physical modes in the generalized Nakagami fading channels. It is shown that even in the AWGN channel, the reduced-mode rate adaptation schemes in $[6,7]$ can have comparable goodput performance as the complete-search rate adaptation scheme in [5].

On the other hand, with the goal of improving energy efficiency, a path-loss based power control scheme was proposed in [9] for the IEEE $802.11 \mathrm{a} / \mathrm{g}$ WLAN. According to the received power of RTS frame, this power control scheme estimates the path loss and then adds the estimated path loss information into the CTS frame. Upon receiving the CTS frame, the user adjusts the transmit power based on the estimated path loss. In [10], the authors proposed a heuristic algorithm for joint link adaptation and power control to improve throughput in wireless packet networks. This work considers the case where the rate and power level 
adjustments are continuous. Besides, transmission power is adjusted by a Kalman-filter based method to achieve successful transmission. The authors in [11] proposed a minimum-energy transmission strategy in the AWGN channel. This algorithm aims to maximize the ratio of the successfully received bits over the energy consumption. The algorithm in [11] can be treated as the energy-optimal link adaptation scheme in the AWGN channel since it completely searches among all the combinations of MCS and power levels. Fewer papers have considered the tradeoff between energy efficiency and goodput in the joint rate and power adaptation mechanism. In the previous work [12], the authors proposed a joint rate and power adaptation algorithm to improve goodput and energy efficiency. The work in [12] considers a simple single-user case where the impact of multiple-user contention is not considered.

This paper focuses on investigating the tradeoff between energy efficiency and goodput in the WLANs, by properly determining the combination of MCS and power level. We propose an efficient channel-driven rate and power adaptation (CDRPA) algorithm in the generalized Nakagami fading channel. The CDRPA algorithm dynamically adjusts the data rate and power according to the signal strength of received CTS frame in an open-loop control fashion. By using the reduced-mode rate selection scheme of [6,7], the CDRPA algorithm has much lower computation complexity than the energy-optimal complete-search scheme [11]. In WLANs, energy efficiency can be traded for goodput by adopting a higher data rate with higher transmission power. Therefore, this paper compares two CDRPA schemes to investigate the tradeoff between energy efficiency and goodput. The power-first CDRPA scheme first selects the transmit power aiming at energy saving, and then determines the data rate for transmission. On the contrary, the rate-first CDRPA scheme has the goal of boosting goodput.

Furthermore, we develop a physical (PHY)/medium access control (MAC) cross-layer analytical method incorporating the impacts of Nakagami fading channel and the carrier sense multiple access (CSMA) MAC protocol. Different from the previous work, this paper considers a practical case where several users contend for the channel. In such a multiuser contention environment, we analyze the goodput and the energy efficiency of two CDRPA schemes and the energy-optimal complete-search adaptation scheme to obtain important insights into the design of joint power and rate adaptation scheme. Moreover, a simple but effective weighted moving-average approach is suggested to predict the channel quality. It will be shown that this channel prediction approach can improve the energy efficiency of CDRPA schemes without degrading goodput.

The rest of this paper is organized as follows. Section 2 introduces the Nakagami fading channel model, the physical and MAC layers of the IEEE 802.11a wireless local area network (WLAN). In Sect. 3, we present the channel-driven rate and power adaptation (CDRPA) algorithm. In Sect. 4, we develop the cross-layer analytical method to evaluate the goodput and energy efficiency of CDRPA schemes in a multiuser contention scenario. Sect. 5 shows the numerical results. Conclusions are given in Sect. 6.

\section{System Model}

\subsection{System Description and Assumptions}

This paper considers the IEEE 802.11a WLAN as an example to evaluate the performance of proposed adaptation algorithm. The IEEE 802.11a WLAN provides eight transmission PHY modes (i.e., eight modulation and coding schemes) with the data rates ranging from 6 to 54 Mbps as listed in Table 1 [13]. 
Table 1 Eight PHY

Rate-dependent parameters

* Bytes per OFDM symbol

\begin{tabular}{lllll}
\hline Mode $\mathrm{m}$ & Data rate & Modulation & Coding rate & $\mathrm{BpS}(\mathrm{m})^{*}$ \\
\hline 1 & $6 \mathrm{Mbps}$ & BPSK & $1 / 2$ & 3 \\
2 & $9 \mathrm{Mbps}$ & BPSK & $3 / 4$ & 4.5 \\
3 & $12 \mathrm{Mbps}$ & QPSK & $1 / 2$ & 6 \\
4 & $18 \mathrm{Mbps}$ & QPSK & $3 / 4$ & 9 \\
5 & $24 \mathrm{Mbps}$ & 16-QAM & $1 / 2$ & 12 \\
6 & $36 \mathrm{Mbps}$ & 16-QAM & $3 / 4$ & 18 \\
7 & $48 \mathrm{Mbps}$ & 64-QAM & $2 / 3$ & 24 \\
8 & $54 \mathrm{Mbps}$ & 64-QAM & $3 / 4$ & 27 \\
\hline
\end{tabular}

(a)

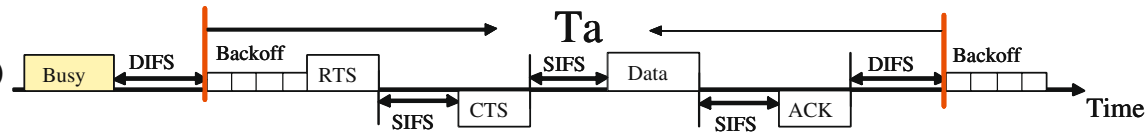

(b)

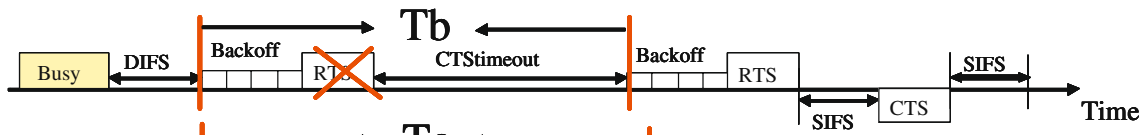

(c)

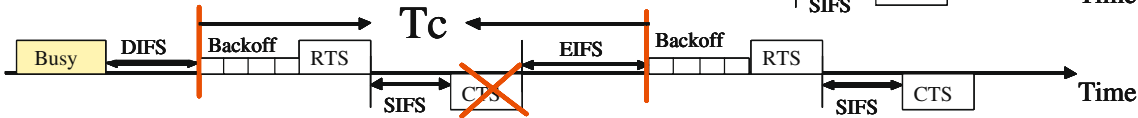

(d)

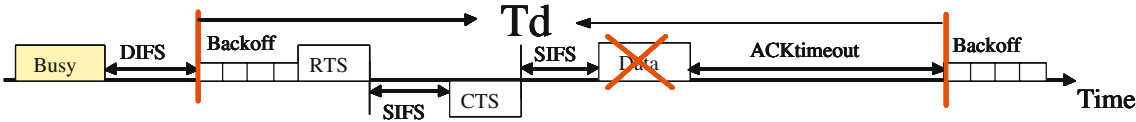

(e)

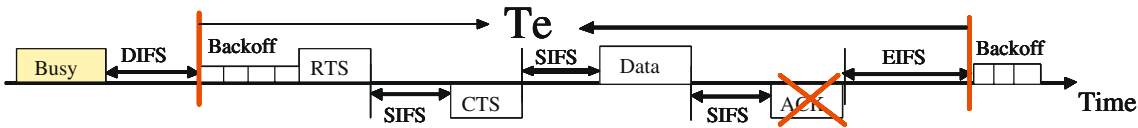

Fig. 1 Possible frame transmission scenarios according to the CSMA MAC protocol with RTS/CTS: (a) successful frame transmission; (b)-(e) erroneous RTS/CTS/data/ACK

In this power-adaptive wireless network, four-way handshaking RTS/CTS mechanism is needed to avoid the hidden node problem. Specifically, the users in a power-adaptive wireless network have different power levels. The user with higher power may interfere with the lower-power frame transmission, since it does not sense the transmission with lower power. The RTS/CTS handshaking at the maximum power level is a simple method to avoid such a hidden node problem. Figure 1 shows five possible frame transmission scenarios according to CSMA/CA MAC protocol with RTS/CTS mechanism. Figure 1a shows the sequence of a successful data transmission, i.e. "RTS-CTS-DATA-ACK". Figure 1b-e respectively represent the unsuccessful frame transmissions due to contention collision of RTS frame or reception error of RTS/CTS/DATA/ACK frame.

\subsection{Generalized Nakagami Fading Channel Model}

In a generalized Nakagami fading channel [14], the probability density function (pdf) of the received signal in the Nakagami fading channel is given as 


$$
p(\alpha)=2\left(\frac{M}{\Omega}\right)^{M} \frac{\alpha^{2 M-1}}{\Gamma(M)} \exp \left(-M \frac{\alpha^{2}}{\Omega}\right),
$$

where $\alpha, M, \Omega=E\left[\alpha^{2}\right], \Gamma(M)$ are the fading amplitude, the Nakagami fading parameter, the local mean power, and the Gamma function, respectively. The received signal-to-noise ratio (SNR) $\gamma$ is then gamma distributed with the pdf $p_{r}(r)$, and [14]

$$
p_{\gamma}(\gamma)=\left(\frac{M}{\bar{\gamma}}\right)^{M} \frac{\gamma^{M-1}}{\Gamma(M)} \exp \left(-M \frac{\gamma}{\bar{\gamma}}\right),
$$

where $\bar{\gamma}$ is the average received SNR.

The Nakagami channel model can describe the multipath fading environment with a lineof-sight (LOS) component. The less the Nakagami shape factor $M$, the severer the fading. The Nakagami channel becomes the Rayleigh fading channel as $M=1$, while the Nakagami fading channel becomes the non-fading AWGN channel as $M \rightarrow \infty$.

\subsection{Channel Transition Probability Matrix}

The fading channel can be described by the Markov chain with several states according to the channel quality. The Gilbert channel model is a well-known one, in which the channel is described by two states - "GOOD and $B A D$ ". In [6], the fading channel is divided into multiple channel states $s_{j}$, each of which is associated with a required $E_{b} / N_{0}$ threshold $\left(\eta_{j}\right)$ for data rate $j=1,2, \ldots, 8$ in the IEEE 802.11a WLAN. That is, the state variable $s_{j}$ represents the event that the received $E_{b} / N_{0} \in\left[\eta_{j}, \eta_{j+1}\right)$.

Let $p_{j k}$ represent the channel transition probability from state $s_{j}$ to $s_{k}$, and $x_{t}$ be the $E_{b} / N_{0}$ at the time instant $t$. Then, $p_{j k}$ can be expressed as

$$
\begin{aligned}
p_{j k} & =P\left(x_{t} \in\left(\eta_{k}, \eta_{k+1}\right) \mid x_{t-\tau} \in\left(\eta_{j}, \eta_{j+1}\right)\right) \\
& =\frac{\int_{\eta_{k}}^{\eta_{k+1}} \int_{\eta_{j}}^{\eta_{j+1}} p\left(\gamma_{t-\tau}, \gamma_{t}\right) d \gamma_{t-\tau} d \gamma_{t}}{\int_{0}^{\infty} \int_{\eta_{j}}^{\eta_{j+1}} p\left(\gamma_{t-\tau}, \gamma_{t}\right) d \gamma_{t-\tau} d \gamma_{t}} .
\end{aligned}
$$

The state transition probability $p_{j k}$ in (3) represents the channel quality fluctuation in a Nakagami fading channel. In Sect.4, this transition probability will be applied to evaluate the goodput and energy efficiency of CDRPA algorithm in the Nakagami fading channels. In a Nakagami fading channel, the joint pdf $p\left(\gamma_{t-\tau}, \gamma_{t}\right)$ of two channel estimations $\gamma_{t-\tau}$ and $\gamma_{t}$ at the time instants $(t-\tau)$ and $t$ can be given as [15]

$$
\begin{aligned}
p\left(\gamma_{t-\tau}, \gamma_{t}\right)= & \frac{1}{(1-\rho) \Gamma(M)}\left(\frac{\gamma_{t-\tau} \gamma_{t}}{\rho}\right)^{(M-1) / 2}\left(\frac{M}{\bar{\gamma}}\right)^{M+1} \\
& \times I_{M-1}\left(\frac{2 M \sqrt{\rho \gamma_{t-\tau} \gamma_{t}}}{(1-\rho) \bar{\gamma}}\right) \exp \left(-M \frac{\left(\gamma_{t-\tau}+\gamma_{t}\right)}{(1-\rho) \bar{\gamma}}\right),
\end{aligned}
$$

where $I_{M-1}(\cdot)$ is the $(M-1)$-th order modified Bessel function of the first kind; $\bar{\gamma}=$ $E\left[\gamma_{t-\tau}\right]=E\left[\gamma_{t}\right]$ is the average received SNR. In (4), $\rho$ is the power correlation coefficient between fading power $\gamma_{t}$ and $\gamma_{t-\tau}$. According to [16], $\rho$ is given by

$$
\rho=\left|\frac{I_{0}\left(\sqrt{\kappa^{2}-\left(2 \pi f_{D} \tau\right)^{2}+j 4 \pi \kappa f_{D} \tau \cos \mu}\right)}{I_{0}(\kappa)}\right|^{2}
$$

In (5), $\kappa$ means the beamwidth parameter, $f_{D}$ stands for the maximum Doppler frequency in $\mathrm{Hz}$, and $\mu$ represents the mean direction of the angle of arrival. 


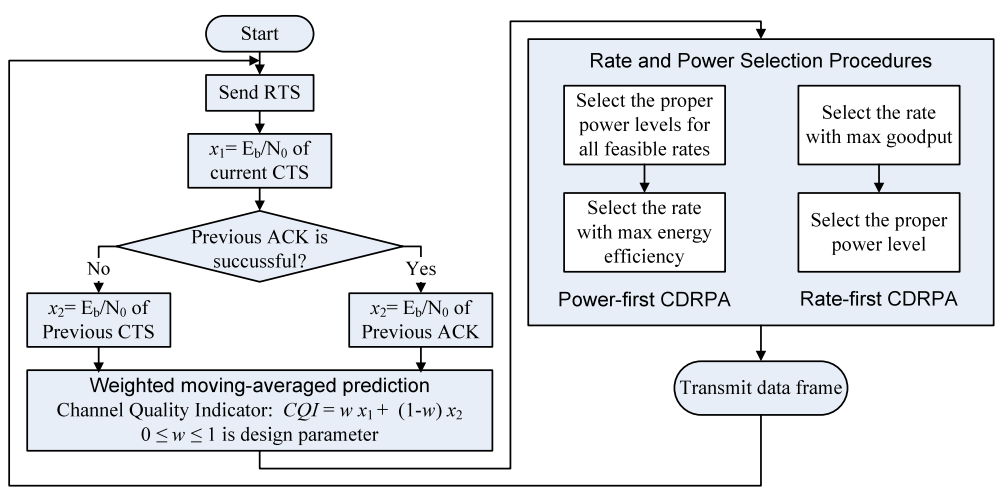

Fig. 2 The flow chart of the CDRPA algorithm with the moving-average channel quality prediction

\section{Channel-Driven Rate and Power Adaptation with Channel Quality Prediction}

This section presents the joint rate and power adaptive CDRPA algorithm. Based on the principle of CDRPA algorithm, we develop two link adaptation schemes: the power-first and the rate-first CDRPA schemes. In this section, we also propose a weighted-average approach to predict the channel quality. Then, the rate and power selection are based on the predicted channel condition.

\subsection{Principle}

The main principle of CDRPA algorithm is to adaptively adjust the transmission rate and power according to the received signal strength $\left(E_{b} / N_{0}\right)$ of CTS frame on an open-loop control basis. In WLANs, the frame transmissions are in the same frequency band, and the forward and reverse links are time-division duplex. In a low-mobility environment, it is reasonable to assume that both links have the similar fading characteristics. In CDRPA, all the control frames (RTS, CTS, and ACK frames) are transmitted at the maximal power $\left(p_{t_{\text {max }}}\right)$ for reliability. Therefore, we can use the received $E_{b} / N_{0}$ of control frame to indicate the channel quality at a given noise power spectra density $N_{0}$. By this open-loop control, we do not need to modify the CTS frame format to include an explicit SNR indicator.

Referring to Fig. 2, the procedures of CDRPA algorithm with the moving-average channel prediction are described in the following:

(I) Channel quality prediction:

We estimate the channel condition according to the following steps:

1. After RTS and CTS frames are successfully exchanged, estimate the received $E_{b} / N_{0}$ of current CTS frame, denoted by $x_{1}$.

2. Let $x_{2}$ be the received $E_{b} / N_{0}$ of ACK frame in the previous data frame transmission. If the ACK frame of previous transmission attempt is not successfully received, let $x_{2}$ be the $E_{b} / N_{0}$ of CTS frame in the previous transmission attempt. Using a weighted moving-average approach, we forecast the channel condition as the channel quality indicator $(C Q I)$

$$
C Q I=w x_{1}+(1-w) x_{2},
$$


where $0 \leq w \leq 1$ is a design factor. In case the RTS frame in the previous transmission attempt is collided or received in error, the transmitter does not receive CTS and ACK frames in the previous transmission attempt. In this situation, we let $w=1$. With the channel quality prediction, the transmit rate and power can be appropriately determined. Therefore, the number of retransmissions and the total energy consumption for transmitting a data frame can be reduced.

(II) Power and rate adaptation:

CDRPA algorithm jointly adjusts the transmit data and power according to the predicted channel quality indicator $C Q I$ obtained by (6). Briefly, CDRPA algorithm calculates the power level for the energy-efficient PHY modes according to the predicted channel quality $C Q I$. Then, following either the most energy-efficient or the highest goodput criteria, CDRPA algorithm selects the data rate from an established reduced-mode table. In the following, we discuss the transmission power and rate selection rules in CDRPA algorithm in more detail.

\subsection{Power and Rate Selection Rules}

\subsubsection{Link-Margin Power Selection}

The transmission power for data frame is determined according to the predicted channel quality $C Q I$ and available link margin. The estimated channel quality $C Q I$ can be calculated by (6), based on the received $E_{b} / N_{0}$ of the CTS and ACK frames. Suppose that $\eta_{r e q}$ is the $E_{b} / N_{0}$ threshold for the selected transmission rate. The available link margin $L_{\text {margin }}$ is defined as

$$
L_{\text {margin }}=C Q I-\eta_{\text {req }} .
$$

In CDRPA algorithm, the transmission power of CTS and ACK frames are fixed at the maximal power level $p_{\text {tmax }}$. Therefore, by the open-loop power control concept, the available link margin $L_{\text {margin }}$ can be treated as how much power can be decreased without causing erroneous reception. Furthermore, the data frame transmission power $p_{t}$ can be determined by

$$
p_{t}=\left\lceil p_{t_{\max }}-L_{\text {margin }}\right\rceil
$$

where $p_{t_{\max }}$ is the maximal transmission power level.

\subsubsection{Reduced-Mode Rate Selection}

One appealing feature of CDRPA algorithm is its reduced-mode rate selection [6,7]. The reduced-mode selection removes the energy-inefficient transmission PHY modes in the rate selection process, thereby increasing goodput and reducing computation cost for the ratepower selection. Consider the IEEE $802.11 \mathrm{a} / \mathrm{g}$ WLAN in the generalized Nakagami fading channel as an example. Table 2a shows the $E_{b} / N_{0}$ thresholds $(\eta)$ for eight transmission PHY modes in the Nakagami fading channel under the requirement of PER $=0.1$ [12]. In the table, it is obvious that the PHY mode with $18 \mathrm{Mbps}$ is energy-inefficient, since its $E_{b} / N_{0}$ threshold is much higher than that for the PHY mode with $24 \mathrm{Mbps}$. Following such a reasoning, we can find that in the Nakagami fading channel, only the PHY modes with respect to 12, 24, 48 , and $54 \mathrm{Mbps}$ should be considered in the rate selection process. Table $2 \mathrm{~b}$ lists these four energy-efficient transmission PHY modes and the corresponding $E_{b} / N_{0}$ ranges for the IEEE 802.11 WLAN in the Nakagami fading channel with $M=1$ and 5. We note that the removed 
Table $2 E_{b} / N_{0}$ requirement at PER $=0.1$ for the IEEE $802.11 \mathrm{a} / \mathrm{g}$ WLAN in the Nakagami fading channel with factors $M=1$ and 5

(a) Eight data rates and the corresponding $E_{b} / N_{0}$ thresholds in the complete-mode rate selection. (The energy-efficient PHY modes are underlined)

\begin{tabular}{|c|c|c|c|c|}
\hline Data Rate (Mbps) & 6 & 9 & 12 & 18 \\
\hline$\eta$ for $m=1$ & 16.25 & 23.80 & $\underline{16.33}$ & 24.20 \\
\hline$\eta$ for $m=5$ & 7.86 & 14.87 & $\underline{7.89}$ & 14.98 \\
\hline Data Rate (Mbps) & 24 & 36 & 48 & 54 \\
\hline$\eta$ for $m=1$ & $\underline{19.11}$ & 28.40 & $\underline{26.90}$ & $\underline{31.88}$ \\
\hline$\eta$ for $m=5$ & 10.62 & 18.90 & $\underline{17.78}$ & 22.56 \\
\hline
\end{tabular}

(b) Four energy-efficient data rates in the reduced-mode rate selection and the required $E_{b} / N_{0}$ ranges

Channel state

Data Rate (Mbps)

$E_{b} / N_{0}$ range for $m=1$

$E_{b} / N_{0}$ range for $m=5$

Channel state

Data Rate (Mbps)

$E_{b} / N_{0}$ range for $m=1$

$E_{b} / N_{0}$ range for $m=5$

\section{$s_{1}$}

12

$[0,19.11)$

$[0,10.62)$

$s_{3}$

48

$[26.90,31.88)$

$[17.78,22.56)$ $s_{2}$

24

[19.11, 26.90)

$[10.62,17.78)$

$s_{4}$

54

$[31.88, \infty)$

$[22.56, \infty)$

PHY modes are energy-inefficient since these modes have lower data rates or higher $E_{b} / N_{0}$ thresholds. In result, although some inefficient PHY modes are removed, the rate adaptation using the reduced-mode table still has a comparable energy efficiency as the complete-mode method.

Based on the principles of CDRPA algorithm presented above, we detail the power-first and rate-first CDRPA schemes as follows. These CDRPA schemes respectively aim to achieve two different goals, maximum energy efficiency or goodput performance.

\subsection{Power-First CDRPA Scheme}

The power-first CDRPA scheme aims at adjusting the transmission power and rate to maximize the energy efficiency of frame transmissions. As shown in Fig. 2, the power and rate selection procedures for the power-first CDRPA scheme are explained as follows:

1. According to the predicted channel quality indicator $C Q I$ obtained by (6), find all the feasible data rates from the reduced-mode table like Table 2 . The feasible data rates are defined as the rates whose $E_{b} / N_{0}$ thresholds are less than the predicted channel quality $C Q I$.

2. For each feasible data rate, determine the power level by (7) and (8); and then calculate the energy efficiency by (16).

3. Select the rate-power combination with the maximal energy efficiency for current data frame transmission.

We explain these steps by the following example. Assume that the maximal transmission power level $p_{t_{\max }}=30 \mathrm{dBm}$, and the estimated channel quality indicator is $C Q I=28 \mathrm{~dB}$. Following step 1, from Table 2, the feasible data rates are 12, 24 or $48 \mathrm{Mbps}$. Next, by (7) and (8), the available link margin for $48 \mathrm{Mbps}$ is $28-26.9=1.1 \mathrm{~dB}$ and the transmission power level is $\lceil 30-1.1\rceil=29 \mathrm{dBm}$. In the same manner, the transmission power levels are deter- 
mined as $21 \mathrm{dBm}$ for $24 \mathrm{Mbps}$ and $19 \mathrm{dBm}$ for $12 \mathrm{Mbps}$, respectively. After computation, we choose the rate-power combination of $(24 \mathrm{Mbps}, 22 \mathrm{dBm})$ for current data frame transmission since it can achieve maximal energy efficiency.

\subsection{Rate-First CDRPA Scheme}

In the power-first CDRPA scheme, goodput performance may be sacrificed to improve energy efficiency. On the contrary, therefore, the goal of rate-first CDRPA scheme is to boost goodput performance. We detail the operation procedures in the following:

1. Based on the predicted channel quality indicator $C Q I$, select the PHY mode with maximal data rate from the feasible PHY modes.

2. According to the link-margin power selection, determine the transmit power for the current data frame by (7) and (8).

We explain these procedures by the same example discussed in Sect.3.3. Following step 1 , the highest feasible data rate is $48 \mathrm{Mbps}$. By (7) and (8), the available link margin is $28-26.9=1.1 \mathrm{~dB}$ and then the transmission power level is $\lceil 30-1.1\rceil=29 \mathrm{dBm}$. Following the procedures, as the channel quality indicator increases, the rate-first CDRPA scheme increases the transmission rate rather than decreases the transmission power. Consequently, the rate-first CDRPA can achieve higher goodput at the given channel condition.

In summary, the power-first CDRPA scheme intends to enhance energy efficiency rather than goodput. On the contrary, the rate-first CDRPA scheme aims to improve goodput. It is expected that the rate-first CDRPA scheme boosts the goodput at the cost of energy consumption. Numerical results will discuss such a tradeoff between energy efficiency and goodput in more detail.

\section{Cross-layer Analysis}

In this section, we develop a PHY/MAC cross-layer analytical model to evaluate the goodput and energy efficiency for the proposed CDRPA algorithm in the Nakagami fading channel. The developed model considers the physical-layer channel effects and the CSMA/CA MAC protocol in a multiuser contention scenario.

\subsection{Goodput}

The goodput is defined as the number of successfully delivered information bits per second. Let $E\left[P_{D}\right]$ be the average delivered information bits, and $E\left[t_{T}\right]$ be the average transmission time for a data frame. Then, the goodput $\mathcal{G}$ is defined as

$$
\mathcal{G}(l, s, m, c, n)=\frac{E\left[P_{D}\right](l, s, m, c, n)}{E\left[t_{T}\right](l, s, m, c, n)},
$$

where $(l, s, m, c, n)$ represent the data payload, the received $E_{b} / N_{0}$, the transmission PHY mode, the number of transmission attempts, and the number of contending users, respectively.

Supposed the number of channel states $N$ and the retry limit $c_{\text {max }}$, the average delivered information bits $E\left[P_{D}\right]$ can be calculated by 


$$
E\left[P_{D}\right](l, s, m, c, n)=\left\{\begin{array}{l}
\left\{P_{S}(l, s, m, n) l\right\}+\left[1-P_{S}(l, s, m, n)\right] \\
\left\{\sum_{k=1}^{N} p_{j k} E\left[P_{D}\right](l, r, m(r), c+1, n)\right\}, \\
\quad \text { for } c \leq c_{\max } \\
0, \quad \text { for } c>c_{\text {max }},
\end{array}\right.
$$

where $P_{S}(l, s, m, n)$ is the successful probability of frame transmission, and $p_{j k}$ is the channel state transition probability as defined in (3). In (10), the first term is the successfully delivered information bits at $c$-th transmission attempt, and the summation means the average successfully delivered bits at $(c+1)$-st transmission attempt. According to the RTS/CTS procedures, a data frame is successfully delivered as long as all the frames in the sequence of "RTS-CTS-DATA-ACK" are successfully received. Therefore, the successful transmission probability $P_{S}(l, s, m, n)$ can be calculated as

$$
\begin{aligned}
P_{S}(l, s, m, n)= & {\left[1-P_{f, R T S}(n, s)\right]\left[1-P_{e, C T S}(s)\right]\left[1-P_{e, \text { data }}(l, s, m)\right] } \\
& \times\left[1-P_{e, A C K}(s)\right],
\end{aligned}
$$

where $P_{f, R T S}(n, s)$ is the failure probability of RTS due to MAC-layer contention collisions or PHY-layer reception error; $P_{e, C T S}(s), P_{e, \text { data }}(l, s, m)$, and $P_{e, A C K}(s)$ are the frame error probability of CTS, data, and ACK frames, respectively. We note that the effect of multiuser contention is included in the probability of $P_{f, R T S}(n, s)$, which is a function of the number of users. Referring to [6] and [17], these probabilities (i.e. $P_{f, R T S}(n, s), P_{e, C T S}(s), P_{e, \text { data }}$ $(l, s, m)$, and $\left.P_{e, A C K}(s)\right)$ can be obtained.

Next, we calculate the average transmission time $E\left[t_{T}\right]$ for a data frame. Since there are five possible frame transmission scenarios according to the RTS/CTS mechanism, the average transmission time $E\left[t_{T}\right]$ can be expressed as

$$
E\left[t_{T}\right](l, s, m, c, n)=\left\{\begin{aligned}
& P_{S}(l, s, m, n) T_{a}+\left[1-P_{S}(l, s, m, n)\right] \\
& \quad\left\{T_{u s}(l, s, m, n)+\sum_{k=1}^{N} p_{j k} E\left[t_{T}\right](l, r, m(r), c+1, n)\right\}, \\
& \text { for } c \leq c_{\max } \\
& 0, \text { for } c>c_{\max },
\end{aligned}\right.
$$

where $T_{a}$ and $T_{u s}(l, s, m, n)$ are respectively the successful transmission duration and the average duration for unsuccessful frame transmission. Let $\overline{T_{b k o f f}}(c)$ be the average backoff window size for the $c$-th transmission attempt, and $T_{\text {data }}(l, m)$ be the transmission time for a data frame with payload size $l$ using PHY mode $m$. Referring to Fig. 1 , the successful transmission duration $T_{a}$ is equal to

$$
T_{a}=\overline{T_{b k o f f}}(c)+T_{R T S}+3 T_{S I F S}+T_{C T S}+T_{\text {data }}(l, m)+T_{A C K}+T_{D I F S},
$$

where $T_{S I F S}$ and $T_{D I F S}$ respectively stand for the durations of a short inter-frame space (IFS), a distributed IFS; $T_{R T S}, T_{C T S}$, and $T_{A C K}$ are the transmission durations for RTS, CTS, and ACK control frames [13]. According to IEEE 802.11a WLAN standard [13], the values of $\overline{T_{b k o f f}}(c)$ and $T_{\text {data }}(l, m)$ can be specified as in [12]. The durations for unsuccessful RTS, CTS, data, and ACK frame transmissions can be expressed as

$$
\left\{\begin{array}{l}
T_{b}=\overline{T_{\text {bkoff }}}(c)+T_{R T S}+T_{C T \text { Stimeout }} \\
T_{c}=\overline{T_{\text {bkoff }}}(c)+T_{R T S}+T_{\text {SIFS }}+T_{C T S}+T_{\text {EIFS }} \\
T_{d}=\overline{T_{\text {bkoff }}}(c)+T_{R T S}+2 T_{S I F S}+T_{C T S}+T_{\text {data }}(l, m)+T_{A C K \text { timeout }} \\
T_{e}=\overline{T_{\text {bkoff }}}(c)+T_{R T S}+3 T_{\text {SIFS }}+T_{C T S}+T_{\text {data }}(l, m)+T_{A C K}+T_{E I F S}
\end{array}\right.
$$


where $T_{E I F S}$ is the duration of an extended IFS, $T_{C T \text { Stimeout }}$ and $T_{A C \text { Ktimeout }}$ represent the durations for CTS and ACK timeout [12,13], respectively. With four possible unsuccessful transmission scenarios in the RTS/CTS procedures, the average duration of unsuccessful transmission $T_{u s}(l, s, m, n)$ can be calculated by

$$
\begin{aligned}
T_{u s}(l, s, m, n)= & \frac{1}{1-P_{S}(l, s, m, n)}\left\{P_{f, R T S}(n, s) T_{b}+\left[1-P_{f, R T S}(n, s)\right] P_{e, C T S}(s) T_{c}\right. \\
& +\left[1-P_{f, R T S}(n, s)\right]\left[1-P_{e, C T S}(s)\right] P_{e, \text { data }}(l, s, m) T_{d} \\
& \left.+\left[1-P_{f, R T S}(n, s)\right]\left[1-P_{e, C T S}(s)\right]\left[1-P_{e, \text { data }}(l, s, m)\right] P_{e, A C K}(s) T_{e}\right\} .
\end{aligned}
$$

\subsection{Energy Efficiency}

The energy efficiency $\zeta$ (in successfully delivered information bits per joule) is defined as the ratio of the average delivered information bits $E\left[P_{D}\right]$ to the total energy consumption $E\left[\varepsilon_{T}\right]$ during a data frame transmission. That is,

$$
\zeta(l, s, m, c, n)=\frac{E\left[P_{D}\right](l, s, m, c, n)}{E\left[\varepsilon_{T}\right](l, s, m, c, n)},
$$

where $E\left[P_{D}\right]$ can be calculated by (10).

Assume that $P_{T}\left(p_{t}\right)$ and $P_{R}$ are the power consumption for a terminal operating in the transmitting and receiving modes as detailed in [12]. With five possible frame transmission scenarios as shown in Fig. 2, the total energy consumption $E\left[\varepsilon_{T}\right](l, s, m, c, n)$ during a data frame transmission can be expressed as

$$
\begin{aligned}
& E\left[\varepsilon_{T}\right](l, s, m, c, n)= \\
& \qquad \begin{array}{c}
P_{S}(l, s, m, n)\left[\left(2 T_{S I F S}+T_{A C K}+T_{D I F S}\right) P_{R}+T_{\text {data }}(l, m) P_{T}\left(p_{t}\right)\right] \\
+P_{f, R T S}(n, s)\left[\left(T_{S I F S}+2 T_{s l o t}\right) P_{R}\right] \\
+\left[1-P_{f, R T S}(n, s)\right] P_{e, C T S}(s)\left[T_{E I F S} P_{R}\right] \\
+\left[1-P_{f, R T S}(n, s)\right]\left[1-P_{e, C T S}(s)\right] P_{e, \text { data }}(l, s, m) \\
\quad\left[\left(T_{S I F S}+T_{A C K \text { timeout }}\right) P_{R}+T_{\text {data }}(l, m) P_{T}\left(p_{t}\right)\right] \\
+\left[1-P_{f, R T S}(n, s)\right]\left[1-P_{e, C T S}(s)\right]\left[1-P_{e, \text { data }}(l, s, m)\right] P_{e, A C K}(s) \\
\quad\left[\left(2 T_{S I F S}+T_{A C K}+T_{E I F S}\right) P_{R}+T_{\text {data }}(l, m) P_{T}\left(p_{t}\right)\right] \\
+\left[1-P_{S}(l, s, m, n)\right]\left\{\sum_{k=1}^{N} p_{j k} E\left[\varepsilon_{T}\right](l, r, m(r), c+1, n)\right\}+\varepsilon_{o}, \text { for } c \leq c_{\text {max }} \\
0, \quad \text { for } c>c_{\text {max }} .
\end{array}
\end{aligned}
$$

In (17), $\varepsilon_{o}=\left(\overline{T_{b k o f f}}(c)+T_{S I F S}+T_{C T S}\right) \cdot P_{R}+T_{R T S} \cdot P_{T}\left(p_{t \max }\right)$ represents the common part of energy consumption for the successful and unsuccessful frame transmissions, including the energy consumption during the backoff period, as well as that for transmitting the RTS frame and waiting for the CTS frame.

\section{Numerical Results}

In this section, we compare goodput and energy efficiency for the proposed CDRPA schemes and the energy-optimal complete-search adaptation scheme [11] in a multiuser contention scenario. The energy-optimal complete-search scheme exhaustively searches all the possible combinations of transmit rates and power levels to maximize the energy efficiency. By contrast, the CDRPA schemes select only among the reduced PHY modes in Table $2 \mathrm{~b}$. We also 
Table 3 System parameters used in numerical results

\begin{tabular}{ll}
\hline Parameters & Value \\
\hline Packet payload & 1,500 bytes \\
RTS & $160 \mathrm{bits}$ \\
CTS & $112 \mathrm{bits}$ \\
ACK & $112 \mathrm{bits}$ \\
Slot time & $9 \mu \mathrm{s}$ \\
SIFS & $16 \mu \mathrm{s}$ \\
DIFS & $34 \mu \mathrm{s}$ \\
EIFS & $118 \mu \mathrm{s}$ \\
Max. Tx. power, $P_{t \max }$ & $30 \mathrm{dBm}$
\end{tabular}

investigate the effect of a simple weighted moving-average channel quality prediction on the CDRPA schemes. Furthermore, we compare the computation complexity between the powerfirst CDRPA scheme and complete searching scheme. The parameters in numerical results are summarized in Table 3. Consider the Doppler spread is $f_{d}=20 \mathrm{~Hz}$ (i.e., the walking speed at $1 \mathrm{~m} / \mathrm{sec}$ ), and there are fifteen transmit power levels ranging from 16 to $30 \mathrm{dBm}$.

\subsection{Comparisons of Goodput and Energy Efficiency}

Figure 3 shows goodput and energy efficiency against the number of contention users in the Rayleigh fading channel. Obviously, due to the increasing contention collisions, the goodput and energy efficiency decrease, as the number of contention users increases. From this figure, we can also investigate the tradeoff between energy efficiency and goodput in the WLANs by comparing the power-first and rate-first CDRPA schemes. Figure 3a shows that the ratefirst CDRPA scheme can achieve better goodput. In Fig. 3b, at the cost of lower goodput, the power-first CDRPA scheme has better energy efficiency. In this example, the rate-first CDRPA scheme has about $21 \%$ higher goodput while having about $11 \%$ lower energy efficiency than the power-first CDRPA scheme. As a result, the rate-first CDRPA scheme can be a good choice if the goodput is the main concern. Noteworthily, even though the CDRPA algorithm adopts a reduced-mode rate selection, the power-first CDRPA scheme has comparable goodput and energy efficiency as the energy-optimal complete-search adaptation scheme.

Figure 4 illustrates goodput and energy efficiency in Nakagami channel with $M=5$. In the same manner, the rate-first CDRPA scheme improves the goodput at the cost of energy efficiency. Comparing Figs. 3 and 4, we can also find that a larger Nakagami fading factor $M$ can lead to higher energy efficiency and goodput. In a Nakagami fading channel, the less the factor $M$, the severer the fading. Therefore, for a larger factor $M$, the users can adopt higher transmit rate and lower power level for frame transmissions, thereby improving goodput and energy efficiency.

\subsection{Impact of Moving-Average Channel Quality Prediction}

This paper suggests a simple weighted moving-average approach to forecast the channel quality. In WLANs, because the channel fading fluctuates in the wireless channel, the frame transmissions with the rate and power adaptation may be received in error. The suggested channel prediction approach helps select a more proper transmission power to save total energy consumption. 

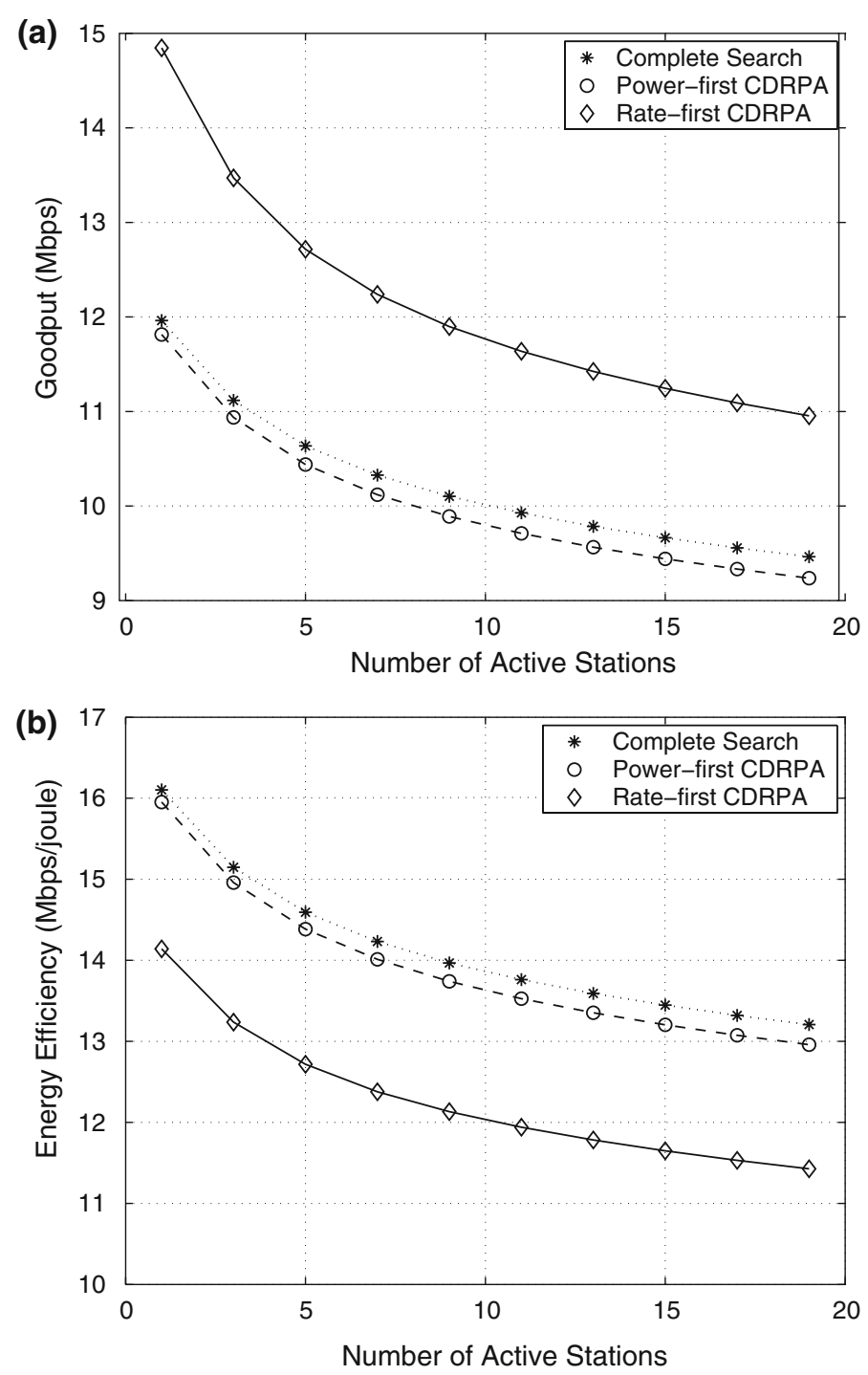

Fig. 3 The goodput and energy efficiency against the number of contending users in Nakagami fading channel with the shape factor $M=1$ : (a) Goodput and (b) energy efficiency

Figure 5 shows the energy efficiency versus the weighting factor $w$ of the moving-average approach. Obviously, one can see that designing an appropriate weighting factor can improve the energy efficiency. In this example, the factor $w$ ranging from 0.4 to 0.6 can improve the energy efficiency of rate-first CDRPA scheme by about $3 \%$ over the case with the factor $w=1$.

Figure 6 illustrates the goodput against the weighting factor $w$. Clearly, referring to Figs. 5 and 6 , the value of $0.4 \leq w \leq 0.6$ can improve the energy efficiency without degrading the goodput. After observing the numerical results, we find that by using the channel prediction approach with the factor $0.4 \leq w \leq 0.6$, the CDRPA algorithm can select the same data rate as the case with $w=1$, but decides a more appropriate power level. Therefore, the 

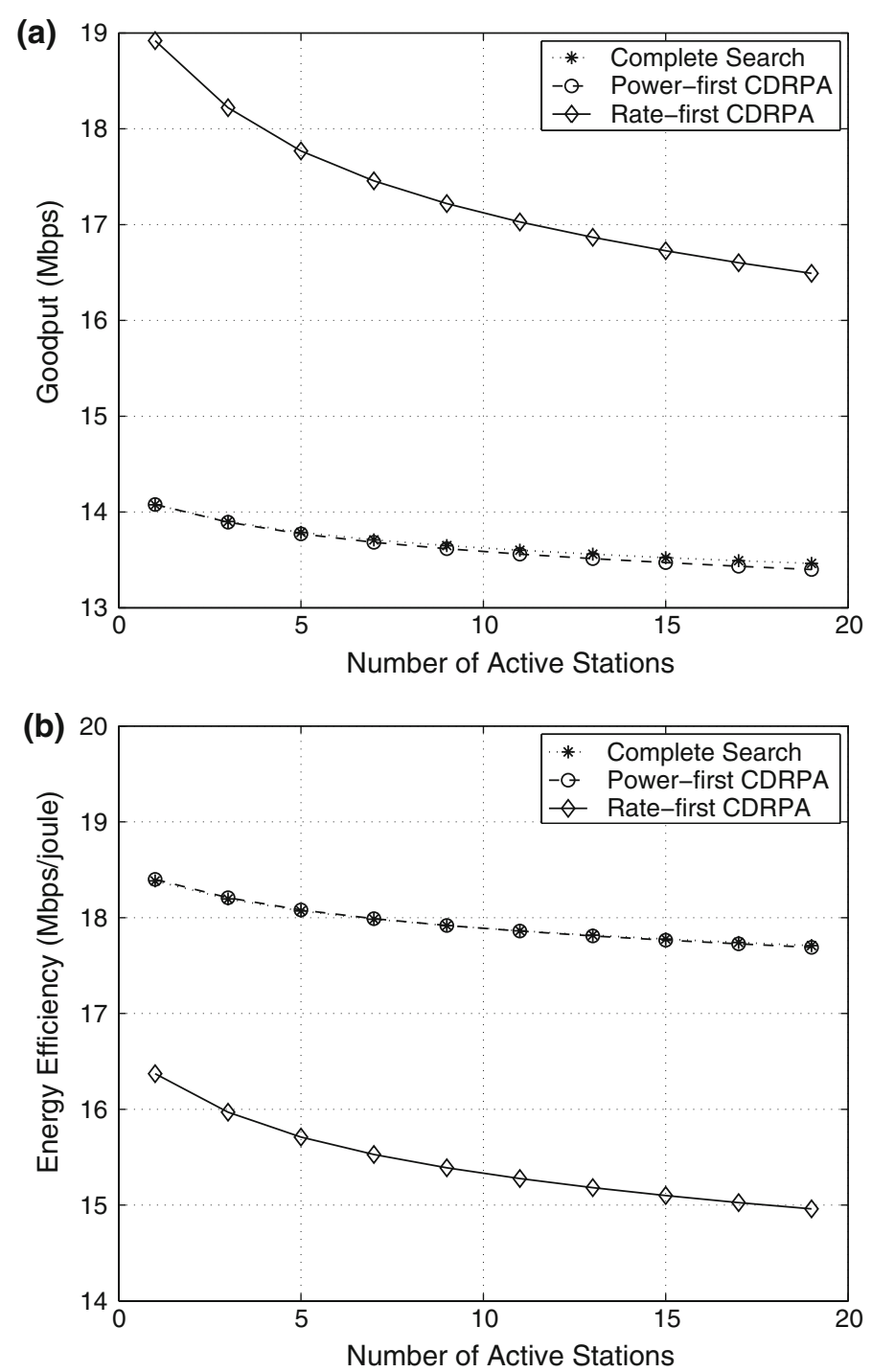

Fig. 4 The goodput and energy efficiency versus the number of contending users in Nakagami fading channel with the shape factor $M=5$ : (a) Goodput and (b) energy efficiency

value of $0.4 \leq w \leq 0.6$ can achieve almost the same goodput but has higher energy efficiency. Figure 6 also shows that the goodput is improved as $w<0.4$. However, this goodput improvement is achieved at a relatively high cost of energy efficiency as shown in Fig. 5.

These figures show that by designing a proper weighting factor $w$, the moving-average channel prediction approach can improve the energy efficiency without goodput degradation. The optimal value of $w$ depends on the communication environment, e.g., user velocity and multipath fading. Other sophisticated channel prediction approaches can be also used to improve the energy efficiency. However, how to design an accurate channel prediction approach to forecast the channel condition is beyond the scope of this paper. 


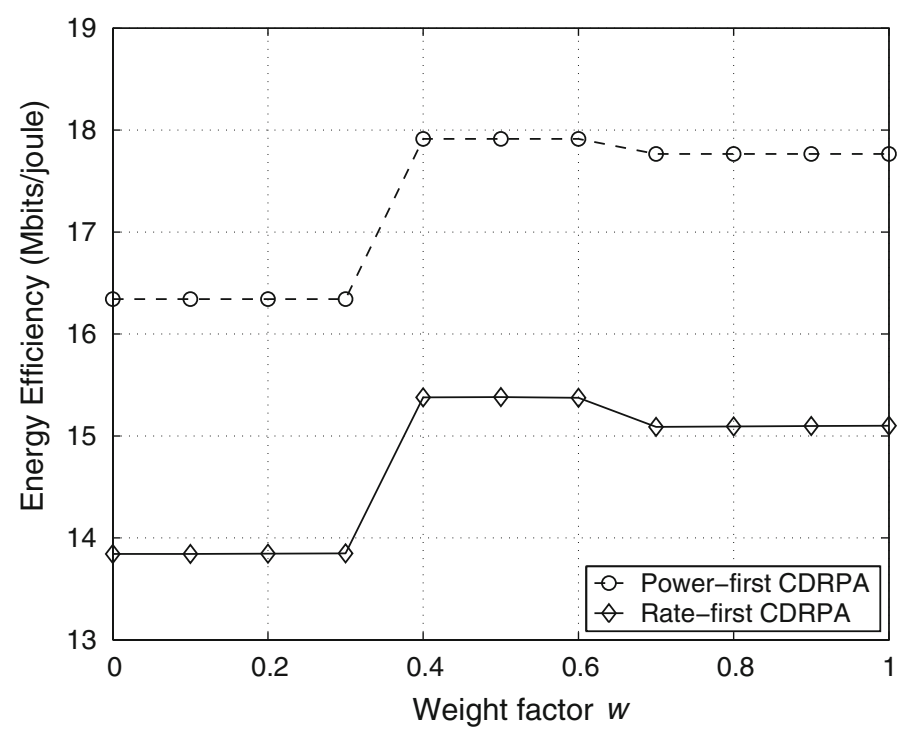

Fig. 5 The energy efficiency of rate-first CDRPA and power-first CDRPA algorithms for various weighting factor $w$, in Nakagami fading channel with $M=5$

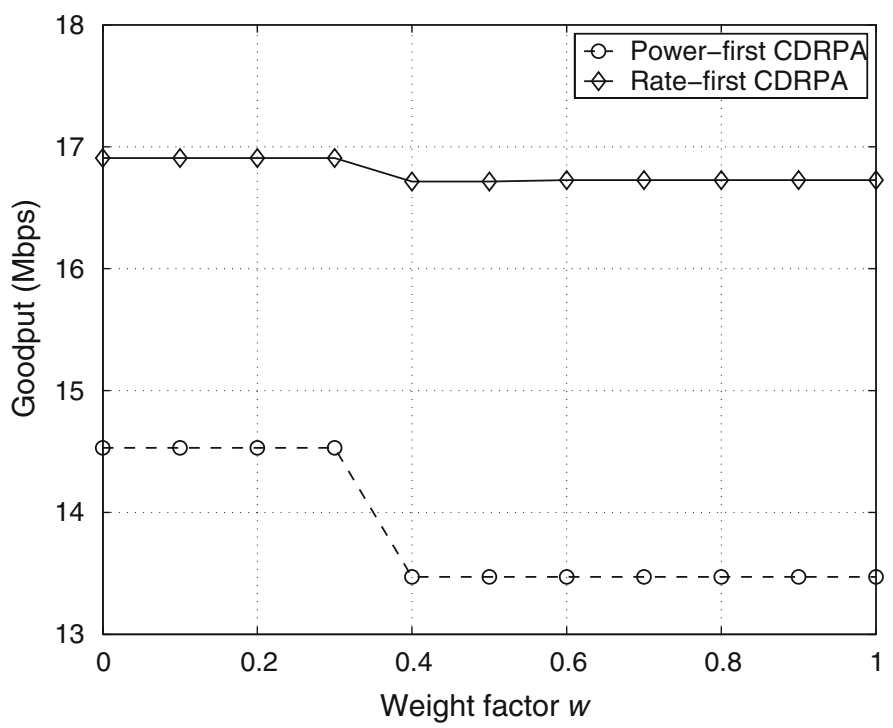

Fig. 6 The goodput of rate-first CDRPA and power-first CDRPA algorithms for various weighting factor $w$, in Nakagami fading channel with $M=5$

\subsection{Computation Complexity}

Table 4 compares the number of possible combinations of data rates and power levels for the energy-optimal complete searching scheme and that for the power-first CDRPA scheme. The 
Table 4 Computation complexity comparison, considering the Rayleigh fading channel

\begin{tabular}{llll}
\hline $\begin{array}{l}\text { Number of } \\
\text { rate-power } \\
\text { candidates }\end{array}$ & Complete search & CDRPA & $\begin{array}{l}\text { Computation } \\
\text { complexity } \\
\text { reduction }(\%)\end{array}$ \\
\hline AWGN $(m=\infty)$ & $8 \times 15$ & 7 & 94.16 \\
Rician $(m=5)$ & $8 \times 15$ & 4 & 96.67 \\
Rayleigh $(m=1)$ & $8 \times 15$ & 4 & 96.67 \\
\hline
\end{tabular}

IEEE 802.11a WLAN provides eight PHY modes for the transmit rate selection. Consider that there are fifteen transmit power levels. The complete searching scheme has to search all possible $(8 \times 15)$ combinations of transmit rates and power levels. By contrast, the power-first CDRPA scheme first determines the power levels for the supported transmit rates according the available link margin, and then select the data rate with the maximal energy efficiency. By the reduced-mode rate selection, the number of supported data rates in CDRPA scheme is less than that in the legacy IEEE 802.11a WLAN, as listed in the table. Therefore, compared to the complete searching method, the computation complexity in CDRPA algorithm can be reduced by $94-97 \%$ in the AWGN and Nakagami channels, respectively.

\section{Conclusions}

This paper has proposed an efficient channel-driven rate and power adaptation (CDRPA) algorithm for WLANs in the generalized Nakagami fading channel. The contributions of this work are described as follows:

- A PHY/MAC cross-layer analytical method is developed to incorporate the impacts of Nakagami fading channel and multiuser contention based on the CSMA MAC protocol.

- We also suggest a simple moving-average channel quality prediction approach, which can improve the energy efficiency of CDRPA schemes without degrading goodput.

- We perform the tradeoff study between energy efficiency and goodput in WLANs for the power-first and rate-first CDRPA schemes as well as the energy-optimal complete-search adaptation scheme. Some important observations are made as follows:

- At the cost of lower goodput, the power-first CDRPA scheme achieves better energy efficiency than the rate-first CDRPA scheme.

- The rate-first CDRPA scheme can be a good choice for systems where the goodput is the major concern.

- Noteworthily, the power-first CDRPA scheme can achieve comparable goodput and energy efficiency as the energy-optimal complete-search scheme, while reducing 94-97\% computation complexity in the rate and power selection.

There are many interesting research topics that can be extended from this work. First, it is worthwhile to further examine the effect of channel estimation accuracy on link adaptation schemes. Second, when the Nakagami fading parameter $M$ is changed dynamically, how to design an effective link adaptation scheme is a challenging task. Third, it is still required to improve the design of the optimal switching points for various modulation and coding schemes in different fading channels. Fourth, we can extend the CDRPA method to consider the delay issue due to retransmission. For example, if a frame has been retransmitted several times, the CDRPA method should adopt higher power and/or lower transmission rate 
for frame transmission to increase successful probability and reduce frame delay. Although there are many rooms for further improvements, the proposed cross-layer analytical model and the CDRPA schemes shed some lights into the optimal cross-layer system design in the future.

Acknowledgements This work was supported in part by the MoE ATU Plan, the Program for Promoting Academic Excellence of Universities (Phase II), the National Science Council, and the Industrial Technology Research Institute under Grant 97W803C, Grant NSC 97-2752-E-009-003-PAE, Grant 97-2221-E-009-097MY3, Grant 97-2221-E-009-099-MY3, Grant 96-2628-E-009-004-MY3, and Grant G1-98006.

\section{References}

1. Kamerman, A., \& Monteban, L. (1997). WaveLAN-II: A high-performance wireless LAN for the unlicensed band. Bell System Technical Journal, 2(33), 118-133.

2. Eckhardt, U., Lenk, M., \& Grell, M. (2003). Transmitter Adjustment Based Transmission Statistics. U.S. Patent 0,086,058, issued on June 19, 2003.

3. Prado, J. D., \& Choi, S. (2004) Method and System for Generating and Updating Transmission Rate for Link Adaptation in IEEE 802.11 WLAN. U.S. Patent 0,017,790, issued on January 29, 2004.

4. Holland, G., Vaidya, N., \& Bahl, P. (2001). A rate-adaptive MAC protocol for multi-hop wireless networks. The ACM Annual International Conference on Mobile Computing and Networking, July 2001 (pp. 236-251).

5. Qiao, D., Choi, S., \& Shin, K. G. (2002). Goodput analysis and link adaptation for IEEE 802.11a wireless LANs. IEEE Transactions on Mobile Computing, 1, 278-292.

6. Wang, L.-C., Lin, Y.-W., \& Liu, W.-C. (2004). Cross-layer goodput analysis for rate adaptive IEEE 802.11a WLAN in the generalized Nakagami fading channel, IEEE International Conference on Communications, June 2004 (pp.2312-2316).

7. Liu, W.-C., Wang, L.-C., \& Lin, Y.-W. (2004). Physical layer effects on the MAC goodput performance for the rate adaptive IEEE 802.11a/g WLAN. IEEE Wireless Communications and Networking Conference, 3, 21-25.

8. Wang, L.-C., Yen, K.-N., Chen, M.-B., Liu, W.-C., Yang, Y.-R., \& Huang, P.-J. (2008). Cross-Layer Rate Adaptation Mechanism for WLAN, U.S. Patent 7,423,970, issued on September 9, 2008.

9. Choi, S., \& Soomro, A. A. (2002). Updating Path Loss Estimation for Power Control and Link Adaptation in IEEE 802.11h WLAN, U.S. Patent 0,168,993, issued on November 14, 2002.

10. Leung, K. K., \& Wang, L.-C. (2002). Integrated link adaptation and power control to improve error and throughput performance in broadband wireless packet network. IEEE Transactions on Wireless Communications, 1, 619-629.

11. Qiao, D., Choi, S., Jain, A., \& Shin, K. G. (2003). MiSer: An optimal low-energy transmission strategy for IEEE $802.11 \mathrm{a} / \mathrm{h}$, The ACM Annual International Conference on Mobile Computing and Networking, September 2003 (pp. 14-19).

12. Wang, L.-C., Liu, W.-C., Chen, A., Yen, K.-N. (2009, May). Joint rate and power adaptation for wireless local area networks in generalized nakagami fading channels, to appear in IEEE Transactions on Vehicular Technology. [Pre-print version is available at http://ieeexplore.ieee.org/iel5/25/4356907/ 04566070.pdf].

13. IEEE 802.11a. (1999, September). Part 11: Wireless LAN medium access control (MAC) and physical layer (PHY) specifications: High-speed physical layer in $5 \mathrm{GHz}$ band, supplement to IEEE 802.11 Standard.

14. Nakagami, M. (1960). Statistical methods in radio wave propagation. Oxford, UK: Pergamon Press.

15. Alouini, M.-S., \& Goldsmith, A. J. (2000, May). Adaptive modulation over Nakagami fading channels. Wireless Personal Communications, 13, 119-143.

16. Filho, J. C. S. S., Yacoub, M. D., \& Fraidenraich, G. (2007). A simple accurate method for generating autocorrelated Nakagami- $m$ envelope sequences. IEEE Communications Letters, 11(3), 231-233.

17. Dong, X. J., \& Varaiya, P. (2005, February). Saturation throughput analysis of IEEE 802.11 wireless LANs for a lossy channel. IEEE Communications Letters, 9(2), 100-102. 


\section{Author Biographies}

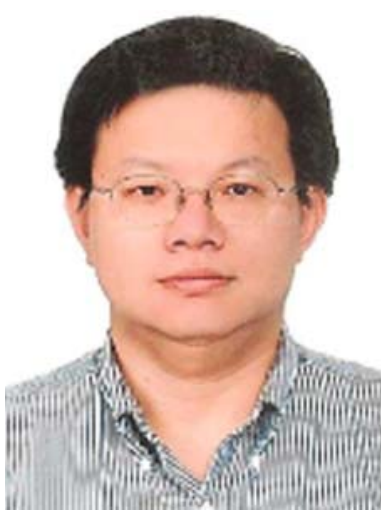

Li-Chun Wang received the B.S. degree from the National ChiaoTung University, Hsinchu, Taiwan, R.O.C., the M.S. degree from the National Taiwan University, Taipei, Taiwan, and the M.Sci. and $\mathrm{Ph} . \mathrm{D}$. degrees from the Georgia Institute of Technology, Atlanta, in 1986, 1988, 1995, and 1996, respectively, all in electrical engineering. Received the BS degree in electrical engineering from the National Chiao-Tung University, Hsinchu, Taiwan, in 1986, the MS degree in electrical engineering from the National Taiwan University, Taipei, in 1988, and the MSc and PhD degrees in electrical engineering from the Georgia Institute of Technology, Atlanta, in 1995 and 1996, respectively. From 1990 to 1992, he was with Chunghwa Telecom. In 1995, he was affiliated with Northern Telecom, Richardson, Texas. From 1996 to 2000, he was with AT\&T Laboratories, where he was a senior technical staff member in the Wireless Communications Research Department. Since August 2000, he has been with the Department of Communication Engineering, National Chiao-Tung University, Taiwan, as an associate professor and has been promoted to a full professor since August 2005. He was a corecipient of the Jack Neubauer Best Paper Award from the IEEE Vehicular Technology Society in 1997. His current research interests include cellular architectures, radio network resource management, cross layer optimization for cooperative, and cognitive wireless networks. He is the holder of three US patents with three more pending. He is a senior member of the IEEE.
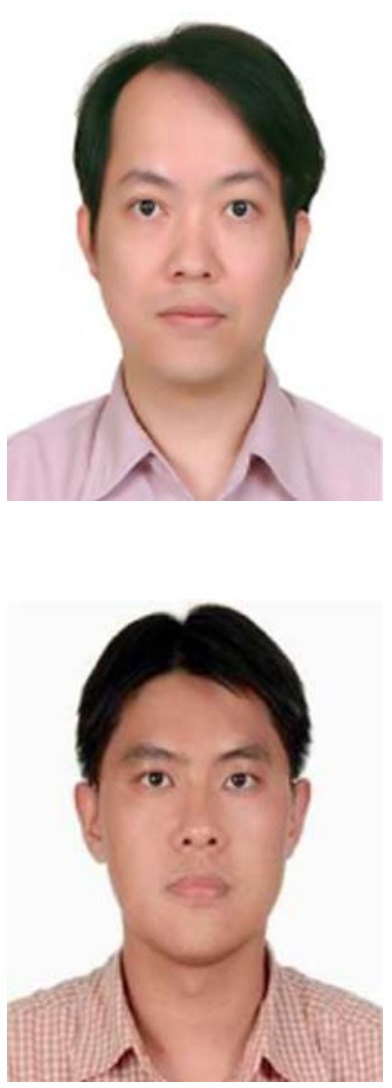

Jane-Hwa Huang received the B.S., M.S., and Ph.D. degrees in electrical engineering from the National Cheng-Kung University, Tainan, Taiwan, R.O.C., in 1994, 1996, and 2003, respectively. He joined the Department of Communication Engineering, National Chiao-Tung University, Taiwan, as a Postdoctoral Researcher from 2004 to January 2006, and a Research Assistant Professor since January 2006. His current research interests are in the areas of wireless networks, wireless multi-hop communications, and radio resource management.

Anderson Chen received the B.S., M.S., and P.h.D. degrees in communication engineering from the National Chaio Tung University, Hsin-Chu, Taiwan, in 1998, 1999, and 2007, respectively. Since October 2008, he is with the Information \& Communications Research Laboratories, Industrial Technology Research Institute of Taiwan, R.O.C. His research interests include wireless networks, cross layer design, and cognitive wireless systems. He is the holder of a US patent with three more pending. 


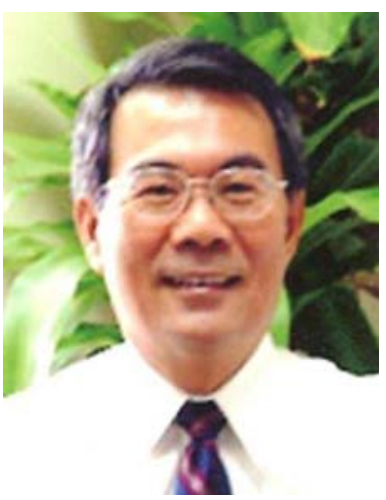

Chung-Ju Chang was born in Taiwan, R.O.C., in August 1950. He received the B.E. and M.E. degrees in electronics engineering from National Chiao-Tung University (NCTU), Hsinchu, Taiwan, in 1972 and 1976, respectively, and the Ph.D. degree in electrical engineering from National Taiwan University (NTU), Taiwan, in 1985. From 1976 to 1988 , he was with Telecommunication Laboratories, Directorate General of Telecommunications, Ministry of Communications, Taiwan, as a Design Engineer, Supervisor, Project Manager, and then Division Director. There, he was involved in designing digital switching system, RAX trunk tester, ISDN user-network interface, and ISDN service and technology trials in Science-Based Industrial Park. In the meantime, he also acted as a Science and Technical Advisor for the Minister of the Ministry of Communications from 1987 to 1989. In 1988, he joined the Faculty of the Department of Communication Engineering and Center for Telecommunications Research, College of Electrical Engineering and Computer Science, National Chiao-Tung University, as an Associate Professor. He has been a Professor since 1993. He was Director of the Institute of Communication Engineering from August 1993 to July 1995, Chairman of Department of Communication Engineering from August 1999 to July 2001, and the Dean of the Research and Development Office from August 2002 to July 2004. Also, he was an Advisor for the Ministry of Education to promote the education of communication science and technologies for colleges and universities in Taiwan during 1995-1999; he is acting as a Committee Member of the Telecommunication Deliberate Body, Taiwan. He serves as Editor for IEEE Communications Magazine and Associate Editor for IEEE Trans. Vehicular Technology. His research interests include performance evaluation, wireless communication networks, and broadband networks. Dr. Chang is a member of the Chinese Institute of Engineers (CIE) and an IEEE Fellow. 